\title{
Effects of passive leg raising on cardiovascular functions as analyzed by fingertip pulse pressure profiles
}

\author{
Kenji Nemoto ${ }^{1}$, Takayo Kaiho ${ }^{2}$, Susumu Ito ${ }^{3}$, Koichi Yoshioka ${ }^{1}$ and Akira Maki ${ }^{1,3 *}$ \\ ${ }^{1}$ Graduate School of Emergency Medical Systems, Kokushikan University, Japan \\ ${ }^{2}$ Depatment of Health Science, Health Science University, Japan \\ ${ }^{3}$ High-Tech Research Centre, Kokushikan University, Japan
}

\begin{abstract}
Objective: This study investigated the efficacy, duration, and possible adverse effects of passive leg raising (PLR) by examining the cardiovascular responses over the entire course of PLR.

Methods/results: Continuous measurements of cardiovascular parameters using a non-invasive finger blood pressure measurement system during PLR were performed on 8 healthy subjects. Blood pressures significantly increased during PLR when legs were raised at $20^{\circ}$, $30^{\circ}$, and $40^{\circ}$ angles. This blood pressure increase was observed to be primarily due to an increase in stroke volume, which remained almost constant until the final 10 min of PLR. No significant differences in cardiovascular parameters between the different PLR angles were observed. The return of the legs to the horizontal position after PLR caused transient changes in cardiovascular parameters, including considerable transient decreases in stroke volume and total peripheral vascular resistance. Relevance of these findings to the cases with deteriorated cardiovascular functions, in which, PLR may cause a severe transient decrease in blood pressure leading to transient cerebral ischemic attack, is discussed.
\end{abstract}

Conclusion: PLR caused increases in blood pressure, primarily because of an increase in stroke volume. A $20^{\circ} \mathrm{PLR}$ angle was sufficient to produce these effects.

Abbreviations: CO: cardiac output; DBP: diastolic blood pressure; HR: heart rate; MBP: mean blood pressure; PLR: passive leg raising; SBP: systolic blood pressure; SV: stroke volume; TPR: total peripheral vascular resistance.

\section{Introduction}

Passive leg raising (PLR) in a supine position has been widely used in primary care for patients with acutely low blood volume [1-3]. The aim of this procedure is to improve blood circulation by facilitating venous return from the lower limbs [1-10]. However, several studies have cast doubts on the efficacy and duration of the effects of PLR [24]. Possibly due to this controversy, in the American Heart Association Guidelines for Cardiopulmonary Resuscitation and Emergency Cardiovascular Care of 2010 [8], PLR was neither recommended nor prohibited for the treatment of hypovolemic shock.

Furthermore, if PLR can positively sustain cardiovascular dynamics, the return of the raised legs to horizontal position may cause "rebound" negative effects, although we could not find previous reports describing any adverse effects of the return of the legs from PLR.

In most of the previous studies that examined PLR efficacy, cardiovascular parameters were observed only at discrete time points during PLR, precluding a view of cardiovascular dynamics over the total time course during and after PLR. Although echocardiogram is useful for estimating cardiovascular parameters, this tool may not be suited for continuous measurements because estimation depends on visual judgments and complex calculations [11]. Because simultaneous measurement of heart rate (HR), cardiac output (CO), total peripheral vascular resistance (TPR), systolic blood pressure (SBP), and diastolic blood pressure (DBP) is difficult, previous studies used only a few of these important cardiovascular parameters to evaluate the dynamic cardiovascular response to PLR. This shortcoming may have led to an incomplete view of the effects of PLR and caused apparent discrepancies in the experimental results between studies.

The purpose of this study was to evaluate the efficacy of PLR by elucidating the entire course of cardiovascular responses to PLR over considerable lengths of duration. In addition, we sought to investigate optimal conditions for effective PLR. We continuously and simultaneously measured multiple parameters of cardiovascular dynamics, including SBP, DBP, HR, CO, and TPR, using a continuous finger blood pressure measurement system, which non-invasively estimated multiple important parameters of cardiovascular dynamics.

Parts of this work have been published in a preliminary form [12].

\section{Materials and methods}

\section{Subjects}

Subjects were 8 healthy adult males with an average age of $32.0 \pm$ 4.6 years, average height of $173.5 \pm 6.1 \mathrm{~cm}$, and an average weight of $76.8 \pm 13.9 \mathrm{~kg}$. Before participating in this study, subjects were fully

Correspondence to: Dr. Akira Maki, Graduate School of Emergency Medical Systems, Kokushikan University, 7-3-1 Nagayama, Tama-shi, Tokyo 206-8515, Japan, Tel: +81-42-339-7279, Fax: +81-42-339-7279, E-mail: maki@kokushikan.ac.jp

Key words: passive leg raising, hypovolemic shock, blood pressure, stroke volume

Received: May 14, 2016; Accepted: June 22, 2016; Published: June 24, 2016 
informed of the purpose, methods, and possible harmful effects of this study. All subjects provided written consent for participation. This study was approved by the Kokushikan University Ethics Committee for Research on Human Subjects.

\section{Measurement device}

A continuous finger blood pressure measurement system (Portapres, Medical Systems B.V.) was used to make continuous and non-invasive measurements of cardiovascular dynamics [13-15]. This system allows one to estimate finger arterial blood pressure with high time resolution by volume clamping finger arteries using optical feedback signals. Based on the method designed by Wesseling and coworkers, brachial arterial blood pressure waves are reconstructed from measured finger blood pressure waves using an assumed standard transfer function from arm to finger. The shape and amplitude of reconstructed brachial arterial pressure waves have been shown to be sufficiently identical to directly measured brachial arterial pressure waves [13-15].

Using calculations based on the mathematical model (Modelflow ${ }^{\circ}$ ) suggested by Wesseling and coworkers [16], Portapres ${ }^{\infty}$ can estimate major cardiovascular parameters, including stroke volume (SV), TPR, SBP, DBP, and HR, which are obtained from reconstructed brachial arterial blood pressure waves.

A finger cuff was fitted on the third finger of the right hand of each subject. At the start of each experiment, SBP and DBP at the right upper arm were manually measured, and the cuff was readjusted when the discrepancy between the reconstructed and manually measured values of either SBP or DBP was more than or equal to $10 \mathrm{mmHg}$.

\section{Measured parameters}

In this study, measurements of SBP, DBP, MBP, HR, SV, CO, and TPR are presented and discussed.

Data obtained using Portapres ${ }^{\oplus}$ were transferred to a personal computer in data table files for a spread sheet application (Excel 2007, Microsoft Corporation). Beat-by-beat data were converted into secondby-second data for further analysis using a data analysis application (KyPlot 5.0, KyensLab Corporation). Data of each parameter were normalized by the average value of the 5 -min period immediately before the start of PLR and expressed as percentages.

\section{Protocol of the PLR experiment}

Initially, each subject rested in the supine position on a horizontal board with a lower section that could be raised to 3 different angles $\left(20^{\circ}, 30^{\circ}\right.$, or $\left.40^{\circ}\right)$. After a 300 -s rest, the subject's legs were passively raised at one of the 3 angles and maintained in the raised position for $600 \mathrm{~s}$. Subsequently, the legs were passively returned to the horizontal position, and the subject rested for $180 \mathrm{~s}$, during which time the recovery processes of cardiovascular functions were monitored. This total 1080 -s sequence was repeated 3 times in a day, with a randomly chosen sequence of the 3 angles (Figure 1). For each subject, the same
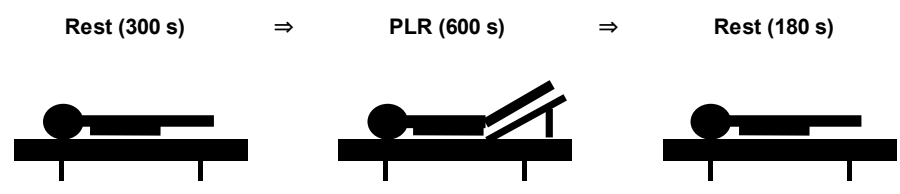

Figure 1. Measurement procedure. One sequence of the PLR experiment consisted of 300 -s rest, 600 -s PLR, and 180 -s post-PLR rest. The sequence was repeated 3 times using a different PLR angle each time $\left(20^{\circ}, 30^{\circ}\right.$, or $\left.40^{\circ}\right)$ in randomized order in 1 day. experiment was repeated 3 times with a different angle sequence for each experiment on different days. Therefore, 24 PLR data sets were obtained from 8 subjects for each of the 3 tested angles. Data points less than 50 or more than 150 after normalization were discarded as artifacts by visual confirmation because they occurred abruptly and briefly, without consistent changes observed for the other measurements. Because data for the $30^{\circ}$ PLR of one subject were observed to have large errors, only 23 sample sets were analyzed for evaluation of the $30^{\circ}$ PLR.

Raising and returning the legs was manually performed by 2 experimenters within $1 \mathrm{~s}$. To minimize psychosomatic influences on cardiovascular dynamics, each subject was kept silent, with eyes masked, and free from unnecessary stimuli except leg raising and returning during the experiment. Room temperature and humidity were maintained at $23.0 \pm 4.6^{\circ} \mathrm{C}$ and $44.0 \pm 5.1 \%$, respectively.

\section{Statistical analysis}

Average values in 3 time sections, 60-120 s (section A), 420-540 $\mathrm{s}$ (section B), and 60-180 s (section C) from the end of PLR were compared with controls (5-min average values before PLR) by $t$-test, and $p$ values $<0.05$ were considered significant.

The effects of the 3 PLR angles on cardiovascular parameters were compared at each section by two-way ANOVA and multiple comparison test (Bonferroni) on KyPlot 5.0.

\section{Results}

Table 1 presents mean values of the measured cardiovascular parameters for $300 \mathrm{~s}$ in the resting state. All mean values are consistent with those of normal adults, and variations between individuals were not excessive.

\section{Time courses of cardiovascular responses to $20^{\circ}, 30^{\circ}$, and $40^{\circ}$ PLRs}

Figure 2 shows the time courses of MBP during the PLR procedure for the 3 tested angles. After a transient up and down response at the start of PLR, MBP increased above that of pre-resting controls and remained elevated during the 600-s leg raise at all PLR angles. The return of the legs to the horizontal position caused a transient decrease in MBP, which subsequently increased again to values higher than those of controls.

As shown in Figure 3, HR tended to gradually decrease during $20^{\circ}$ and $30^{\circ}$ PLR and became significantly low in section B of the $20^{\circ}$ PLR. During the $40^{\circ} \mathrm{PLR}$ response, HR showed no significant change during PLR. Just after the return of the legs, a transient increase in HR lasting approximately $1 \mathrm{~min}$ was observed for all 3 PLR angles. HR remained low for the $20^{\circ} \mathrm{PLR}$, returned to pre-PLR levels for the $30^{\circ} \mathrm{PLR}$, and remained slightly higher than pre-PLR controls in the $40^{\circ}$ PLR.

Table 1. Resting values of cardiovascular parameters.

\begin{tabular}{|l|c|c|}
\hline Cardiovascular Parameter & Mean & SD \\
\hline Systolic Blood Pressure (mmHg) & 122.2 & 6.0 \\
\hline Diastolic Blood Pressure (mmHg) & 67.7 & 5.6 \\
\hline Mean Blood Pressure (mmHg) & 82.9 & 5.8 \\
\hline Heart Rate (bpm) & 55.6 & 6.1 \\
\hline Stroke Volume (mL) & 86 & 7.2 \\
\hline Cardiac Output (L/min) & 4.77 & 0.79 \\
\hline Total Peripheral Resistance (MU) & 1.08 & 0.2 \\
\hline
\end{tabular}

300-s measurements taken immediately before PLR

$\mathrm{N}=72 ; 8$ subjects $\times 3$ repetitions 


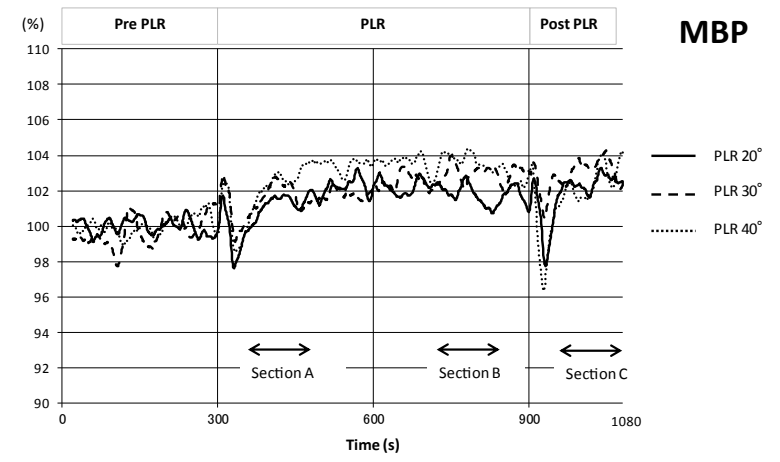

Figure 2. Time course of mean blood pressure changes $(n=24)$ during one sequence of the PLR experiment (shown as normalized values) for 3 different angles $\left(20^{\circ}, 30^{\circ}\right.$, and $\left.40^{\circ}\right)$ Data were plotted after high-cut smoothing.

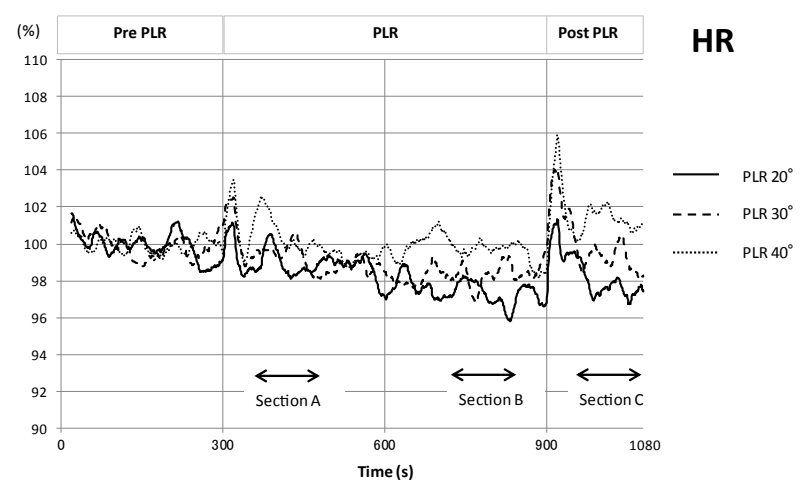

Figure 3. Time course of heart rate changes during one sequence of the PLR experiment. Mean values ( $\mathrm{n}=24$; shown as normalized values) of 3 different PLR angles $\left(20^{\circ}, 30^{\circ}\right.$, and $40^{\circ}$ ) were plotted after high-cut smoothing.

SV immediately increased at the start of PLR to approximately $104 \%$ of pre-PLR controls and decreased immediately upon the return of the legs to the resting position for all 3 PLR angles. The post-PLR SV was almost same as the pre-PLR SV for the $20^{\circ}$ PLR, but decreased to slightly below that of pre-PLR controls for the $30^{\circ}$ and $40^{\circ}$ angle PLRs (Figure 4).

CO increased to approximately $103 \%$ of the pre-PLR control during the first $300 \mathrm{~s}$ of PLR for all 3 PLR angles. For the $20^{\circ}$ and $30^{\circ}$ PLRs, CO gradually decreased in the later $300 \mathrm{~s}$, and the return of the legs to the resting position caused a further decrease compared with that in the pre-PLR controls. For the $40^{\circ} \mathrm{PLR}, \mathrm{CO}$ remained relatively high even later in the $300 \mathrm{~s}$ time course of PLR, and returned to prePLR control levels after a transient increase upon returning of the legs to the resting position (Figure 5).

TPR in all three angle PLRs decreased to approximately $97 \%$ of pre-control values at the start of PLR and gradually increased during PLR to levels slightly higher than pre-PLR controls at the end of PLR. For all 3 angles, TPR increased again after a transient fall upon return of the legs to the resting position (Figure 6).

Comparison of average values of cardiovascular parameters at each section with control values and between the 3 PLR angles

Table 2 shows the average values of cardiovascular parameters at
3 sections for the 3 PLR angles normalized by pre-PLR control values together with their standard errors and $p$-values by $t$-test.

At section A, SBP, MBP, SV, and CO significantly increased for all 3 angles. DBP significantly increased in section A for the $30^{\circ}$ and $40^{\circ}$ PLRs. TPRs were slightly below 100 at section A for all angles, but the differences from controls were not significant.

SBP significantly increased in section B for all angles. DBP for the $30^{\circ}$ PLR and MBP for the $0^{\circ}$ and $40^{\circ}$ PLRs significantly increased. HR significantly decreased for the $20^{\circ}$ PLR, but no significant change was

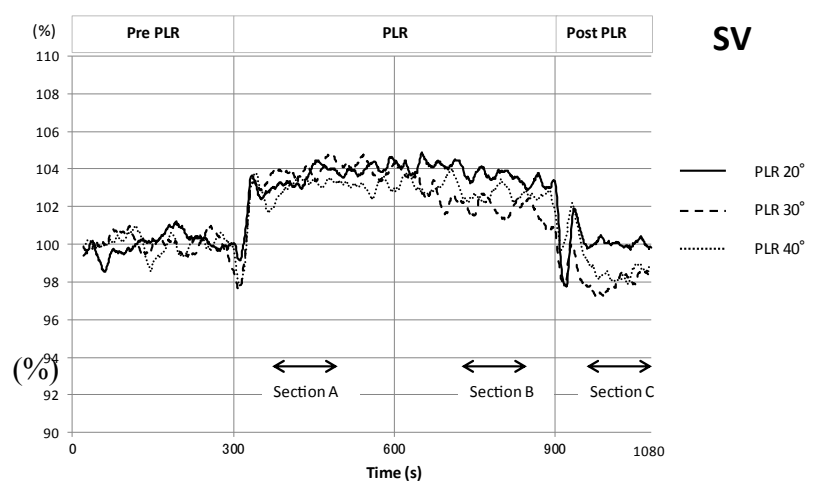

Figure 4. Time course of stroke volume changes during one sequence of the PLR experiment. Analysis and presentation scheme is the same as in Figure 3.

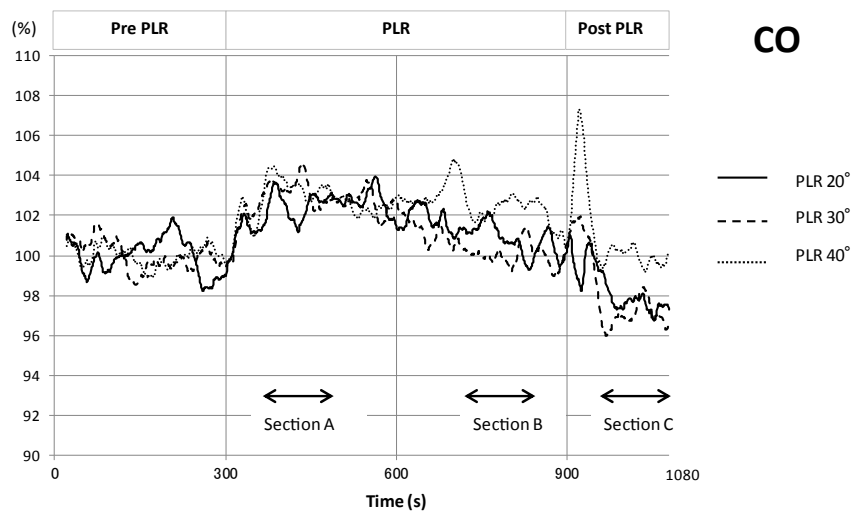

Figure 5. Time course of cardiac output changes during one sequence of the PLR experiment. Analysis and presentation scheme is the same as in Figure 3.

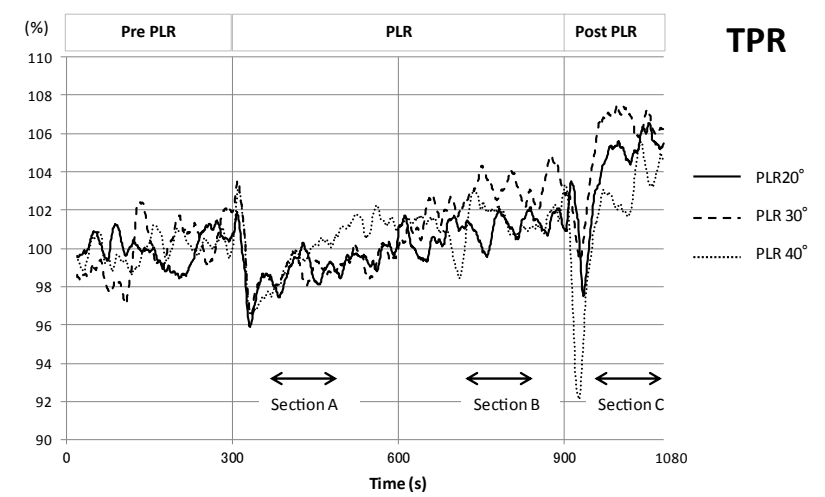

Figure 6. Time course of total peripheral vascular resistance changes during one sequence of the PLR experiment. Analysis and presentation scheme is the same as in Figure 3. 
Table 2. Mean values of parameters for each section normalized by pre-PLR values.

\begin{tabular}{|c|c|c|c|c|c|c|c|c|c|c|}
\hline & \multirow[t]{2}{*}{ PLR angle } & \multicolumn{3}{|c|}{ Section A } & \multicolumn{3}{|c|}{ Section B } & \multicolumn{3}{|c|}{ Section C } \\
\hline & & Mean & SEM & $p$-value & Mean & SEM & $p$-value & Mean & SEM & $p$-value \\
\hline \multirow[t]{3}{*}{ SBP } & $20^{\circ}$ & $102.3^{* *}$ & 0.73 & 0.004 & $104.2 * * *$ & 0.89 & $<0.0001$ & 103 & 1.09 & 0.010 \\
\hline & $30^{\circ}$ & $103.6 * * *$ & 0.75 & $<0.0001$ & $103.8^{* * *}$ & 0.64 & $<0.0001$ & $102.6^{* * *}$ & 0.67 & 0.0008 \\
\hline & $40^{\circ}$ & $104.0 * * *$ & 0.80 & $<0.0001$ & $105.5^{* * *}$ & 1.10 & $<0.0001$ & $103.3^{* *}$ & 1.06 & 0.005 \\
\hline \multirow[t]{3}{*}{ DBP } & $20^{\circ}$ & 100.9 & 0.68 & 0.198 & 101.5 & 0.80 & 0.083 & $102.7^{*}$ & 1.10 & 0.021 \\
\hline & $30^{\circ}$ & $102.2 *$ & 0.93 & 0.028 & $103.5^{* *}$ & 1.02 & 0.003 & $104.1^{* *}$ & 1.25 & 0.003 \\
\hline & $40^{\circ}$ & $102.6 * *$ & 0.73 & 0.002 & $103.8 * * *$ & 0.81 & $\leq 0.0001$ & $103.0 * *$ & 0.95 & 0.005 \\
\hline \multirow[t]{3}{*}{ MBP } & $20^{\circ}$ & $101.4 *$ & 0.62 & 0.034 & $101.7^{*}$ & 0.75 & 0.034 & $102.5^{*}$ & 1.00 & 0.019 \\
\hline & $30^{\circ}$ & 101.7 & 0.77 & 0.035 & 102.0 & 1.32 & 0.145 & 102.5 & 1.44 & 0.098 \\
\hline & $40^{\circ}$ & $102.7 * *$ & 0.64 & 0.0003 & $103.7 * * *$ & 0.65 & $<0.0001$ & $102.7^{* *}$ & 0.86 & 0.004 \\
\hline \multirow[t]{3}{*}{ HR } & $20^{\circ}$ & 99.0 & 0.48 & 0.050 & $97.3^{* * *}$ & 0.73 & 0.0009 & $97.3^{*}$ & 0.90 & 0.011 \\
\hline & $30^{\circ}$ & 100.0 & 0.79 & 0.993 & 99.8 & 1.12 & 0.860 & 100.0 & 0.85 & 0.989 \\
\hline & $40^{\circ}$ & 100.4 & 0.80 & 0.6000 & 99.8 & 1.04 & 0.833 & 101.4 & 1.14 & 0.218 \\
\hline \multirow[t]{3}{*}{ SV } & $20^{\circ}$ & $103.6 * * *$ & 0.84 & 0.003 & $103.6^{* *}$ & 0.96 & 0.001 & 100.2 & 0.96 & 0.872 \\
\hline & $30^{\circ}$ & $102.9^{*}$ & 1.07 & 0.013 & 102.0 & 1.23 & 0.120 & $97.3^{*}$ & 0.97 & 0.012 \\
\hline & $40^{\circ}$ & $103.1^{* *}$ & 1.03 & 0.006 & $102.6^{*}$ & 1.26 & 0.048 & 98.5 & 1.30 & 0.251 \\
\hline \multirow[t]{3}{*}{$\mathrm{CO}$} & $20^{\circ}$ & $102.5^{*}$ & 0.94 & 0.015 & 100.8 & 1.15 & 0.474 & $97.5^{*}$ & 1.12 & 0.038 \\
\hline & $30^{\circ}$ & $103.7 * *$ & 1.26 & 0.008 & 100.6 & 1.17 & 0.604 & 98.0 & 1.17 & 0.104 \\
\hline & $40^{\circ}$ & $103.6^{*}$ & 1.46 & 0.023 & 102.4 & 1.57 & 0.141 & 100.0 & 1.77 & 0.994 \\
\hline \multirow[t]{3}{*}{ TPR } & $20^{\circ}$ & 98.8 & 0.96 & 0.240 & 101.1 & 1.17 & 0.361 & $105.4 * *$ & 1.60 & 0.002 \\
\hline & $30^{\circ}$ & 99.0 & 1.17 & 0.393 & 103.2 & 1.56 & 0.053 & $106.7 * * *$ & 1.75 & 0.009 \\
\hline & $40^{\circ}$ & 99.5 & 1.49 & 0.757 & 101.8 & 1.73 & 0.316 & 103.4 & 1.89 & 0.082 \\
\hline
\end{tabular}

$p$-values were the results of pared $t$ test with pre-PLR resting values. Multiple comparisons (Bonferroni) between different angles showed a significant difference only for CO in section C between the $20^{\circ}$ and $40^{\circ}$ PLRs $(p<0.05) . \mathrm{n}=24$

$* p<0.05 ; * *<<0.01 ; * * * p<0.001$

SBP: systolic blood pressure; DBP: diastolic blood pressure; MBP: mean blood pressure; HR: heart rate; SV: stroke volume; CO: cardiac output; TPR: total peripheral vascular resistance. section A: $120 \mathrm{~s}$ section begins at $60 \mathrm{~s}$ of PLR; section B: $120 \mathrm{~s}$ section begins at $420 \mathrm{~s}$ of PLR; section C: $120 \mathrm{~s}$ section begins at 60 of post-PLR resting.

observed for the other PLR angles. In contrast to section A, TPR was greater than 100 but not significantly different from controls.

In section $\mathrm{C}, \mathrm{SBP}$ and DBP significantly increased for all PLR angles. Increases in MBP were significant only for the $20^{\circ}$ and $30^{\circ} \mathrm{PLR}$ angles. TPR significantly increased for the $20^{\circ}$ and $30^{\circ}$ PLRs. HR for the $20^{\circ}$, SV for the $30^{\circ}$ and CO for the $20^{\circ}$ PLR significantly decreased.

Comparing the effect of different PLR angles on cardiovascular parameters, two-way ANOVA and multiple comparisons revealed a significant difference only for $\mathrm{CO}$ in section $\mathrm{C}$ between the $20^{\circ}$ and $40^{\circ}$ PLRs $(p<0.05)$.

\section{Discussion}

\section{Effects of PLR on cardiovascular parameters and their duration}

In this study, the effects of PLR in healthy subjects on HR, SV, CO, and TPR in addition to SBP, DBP, and MBP were evaluated using a non-invasive continuous measurement system (Portapres ${ }^{\circledR}$ ).

Previous studies on PLR in healthy subjects reported transient changes of cardiovascular dynamics by PLR, but failed to show significant lasting effects of PLR [4-7]. In contrast, the present study revealed significant sustained effects on cardiovascular parameters even at $10 \mathrm{~min}$ after the start of PLR. This discrepancy may be explained from the methodological differences between studies. Other studies evaluated the effects of PLR at discrete time points, while we used averages of continuous measurements for statistical analysis, which enabled us to obtain more stable data to demonstrate the significance of effects that may otherwise be obscured by background fluctuations.
Although a previous study estimated that $150 \mathrm{~mL}$ of blood was moved from the legs to the body by PLR [17], another study reported that the maximal cardiovascular response occurred within $1 \mathrm{~min}$ of the start of PLR and decayed in a short period of time in mechanically ventilated patients [18]. These results depended on discrete time point measurements and pathological states of patients. Therefore, the transferability of their findings to the general population may be unclear.

Out of the parameters observed in this study, SV showed the largest and most stable sustained increase during the entire PLR time course. SV then promptly returned to the pre-PLR values at the end of PLR. It can be believed that this increase was primarily a passive response to the increase in preload due to PLR, which increases central venous pressure, resulting in increased right ventricular output and subsequently that of left ventricular preload. This increase in SV presumably caused increases in the other parameters, including $\mathrm{CO}$, SBP and DBP, during PLR. Wong et al. [1] reported that subjects from whom $500 \mathrm{~mL}$ blood was extracted before PLR showed a significantly greater increase in cardiac index than control subjects. These results, together with our present observations, suggest that PLR may be an effective procedure for patients with relatively normal cardiac functions who are in hypovolemic shock, but not for patients with decreased cardiac functions such as heart failure or old age.

HR tended to decrease in response to PLR. The decrease was particularly significant in section $B$ for the $20^{\circ}$ PLR. In normal subjects, strong homeostatic mechanisms work to maintain constant blood flow to vital organs; the increase in SV caused by PLR may induce a blood pressure increase that is sensed by carotid or cardiopulmonary baroreceptors, and thus, decreases HR through a negative feedback system [19-21]. The decrease in HR during PLR observed in this 
study can, therefore, be interpreted as the result of a compensatory reflex evoked by the increased SV in response to PLR. The lack of HR decrease followed by its increase for the $40^{\circ}$ PLR angle may be due to the uncomfortably high position of the legs because of the large PLR angle.

TPR is another important parameter of cardiovascular function. Although TPR gradually increased after a transient decrease at the start of PLR, no significant changes in TPR were observed at any time for any of the PLR angles. This TPR response to PLR appeared to be transient and compensatory to the rapid increase in SV, which decayed in a relatively short time and caused no significant differences at any time.

$\mathrm{CO}$ is defined as the product of SV and HR. A significant increase in CO during the PLR time course was observed only in the early phase (section A). This increase was probably because the continuing increase in SV dominated over the slight decrease in HR in this time period, while the further decrease in HR in section B cancelled the increased $\mathrm{SV}$, resulting in the gradual decline in CO.

\section{Effects of returning the legs to the horizontal resting position}

In an actual clinical situation, the abrupt release of PLR may occur, for example, when moving a patient from an ambulance to a hospital bed. In this study, considerable changes in cardiovascular dynamics, including some possible adverse ones, were observed upon returning the legs to the horizontal resting position. Blood pressures tended to remain elevated after the return of the legs to the resting position, except for a transient drop lasting several seconds. This second increase in blood pressure appeared to be primarily caused by the increase in TPR, which was probably due to a compensatory reflex to the abrupt decrease in SV by the return of the legs to a horizontal position.

TPR is believed to be maintained by the contraction of arterioles under the control of the sympathetic nervous system. Therefore, in states of deteriorated autonomic nervous system function, such as diabetes mellitus or Shy-Drager syndrome, abrupt returning of legs may result in a severe blood pressure drop.

\section{PLR Angle}

In this study, the PLR angles were chosen considering the admissible range of angles for practical use. Of these, $20^{\circ}$ is the standard angle in Japan for PLR, and $40^{\circ}$ was the maximal angle possible for safe management of subjects.

Of the 3 angles tested, no significant difference in the effects of PLR on cardiovascular parameters was observed except between $20^{\circ}$ and $40^{\circ}$ for the effect on HR after returning the legs to the horizontal position. In particular, SV, which showed the most consistent responses of observed parameters, showed no significant difference in section A or $B$ between the 3 angles tested. Furthermore, the possible adverse effects of the return of the legs to horizontal position appeared to be greater for the larger PLR angles. From these considerations, we regard the $20^{\circ}$ PLR angle as the most suitable for actual clinical purposes.

\section{Limitations}

While the present experiments on healthy adult subjects enabled us to evaluate standard cardiovascular responses to PLR, these data may be insufficient to estimate PLR efficacy in hypovolemic patients with deteriorated cardiovascular functions. However, for such patients, PLR would be expected to be more effective because of the lack of strong homeostatic control present in healthy subjects.
Volume clamp blood pressure measurement is a rather sophisticated method that sometimes becomes unstable; however, we acquired stable, consistent measurements by careful fitting of the cuff. Several papers assured the reliability of measurements using Portapres ${ }^{\oplus}$, with cautions regarding its limitations [13-15]. An improved finger measurement system, Nexfin, recently became available [7]. Re-assessment of the results of this study using such a system would be necessary in future work.

\section{Conclusions}

PLR caused increases in blood pressure, primarily because of an increase in stroke volume. We regard the $20^{\circ}$ PLR angle as the most suitable for actual clinical purposes. The possible adverse effects of the return of the legs to horizontal position appeared to be greater for the larger PLR angles.

\section{Acknowledgements}

We are grateful to Professor Tsuyoshi Watanabe for supports and advices in this study. SI, KY and AM were funded by Institute of Health, Physical Education and Sports Science, Kokushikan University. KN was funded by Physical Education and Sports Science Association, Kokushikan University.

\section{Competing of interest}

The authors declare that they have no competing interests.

\section{References}

1. Wong DH, O'Connor D, Tremper KK, Zaccari J, Thompson P, et al. (1989) Changes in cardiac output after acute blood loss and position change in man. Crit Care Med 17: 979-983. [Crossref]

2. Wong DH, Tremper KK, Zaccari J, Hajduczek J, Konchigeri HN, et al. (1988) Acute cardiovascular response to passive leg raising. Crit Care Med 16: 123-125. [Crossref]

3. Monnet X, Teboul JL (2008) Passive leg raising. Intensive Care Med 34: 659-663. [Crossref]

4. Gaffney FA, Bastian BC, Thal ER, Atkins JM, Blomqvist CG (1982) Passive leg raising does not produce a significant or sustained autotransfusion effect. J Trauma 22: 190193. [Crossref]

5. Yoshioka A, Nishimura K, Seki K, Onodera N (2010) Effects of passive leg raising on cross sectional area of inferior vena cava and stroke volume. Kawasaki Med Welfare J 19: 285-290.

6. Kamran H, Salciccioli L, Gusenburg J, Kazmi H, Ko EH, et al. (2009) The effects of passive leg raising on arterial wave reflection in healthy adults. Blood Press Monit14: 202-207. [Crossref]

7. Sipkens LM, Treskes K, Ariese-Beldman K, Veerman DP, Boer C (2011) Application of Nexfin noninvasive beat-to-beat arterial blood pressure monitoring autonomic function testing. Blood Press Monit 16: 246-251.

8. Markenson D, Ferguson JD, Chameides L, Cassan P, Chung K-L, et al. (2010) Part 13: first aid: 2010 American Heart Association and American Red Cross International Consensus on First Aid Science With Treatment Recommendations. Circulation 122 (suppl): S582-S605. [Crossref]

9. Boulain T, Achard JM, Teboul JL, Richard C, Perrotin D, et al. (2002) Changes in BP induced by passive leg raising predict response to fluid loading in critically ill patients. Chest 121: 1245-1252. [Crossref]

10. John G, Peter JV, Pichamuthu K, Chacko B (2011) Shock for the Internist. Indian J Med 1: 4-23.

11. Marik PE, Monnet X, Teboul JL (2011) Hemodynamic parameters to guide fluid therapy. Ann Intensive Care 1: 1. [Crossref]

12. Nemoto K, Kaiho T, Ito S, Yoshioka K, Maki A (2015) Effects of passive leg raising on stroke volume and blood pressure (in Japanese). Taiiku Sports Kagaku Kenkyu2015 15:63-73.

13. Bos WJ, van Goudoever J, van Montfrans GA, van den Meiracker AH, Wesseling KH 
(1996) Reconstruction of brachial artery pressure from noninvasive finger pressure measurements. Circulation 94: 1870-1875. [Crossref]

14. Gizdulich P, Imholz BP, van den Meiracker AH, Parati G, Wesseling KH (1996) Finapres tracking of systolic pressure and baroreflex sensitivity improved by waveform filtering. J Hypertens 14: 243-250. [Crossref]

15. Gizdulich P, Prentza A, Wesseling KH (1997) Models of brachial to finger pulse wave distortion and pressure decrement. Cardiovasc Res 33: 698-705. [Crossref]

16. Wesseling KH, Jansen JR, Settels JJ, Schreuder JJ (1993) Computation of aortic flow from pressure in humans using a nonlinear, three-element model. J ApplPhysiol(1985) 74: 2566-2573. [Crossref]

17. Rutlen DL, Wackers FJ, Zaret BL (1981) Radionuclide assessment of peripheral intravascular capacity: a technique to measure intravascular volume changes in the capacitance circulation in man. Circulation 64: 146-152. [Crossref]
18. Monnet X, Rienzo M, Osman D, Anguel N, Richard C, et al. (2006) Passive leg raising predicts fluid responsiveness in the critically ill. Crit Care Med 34: 1402-1407. [Crossref]

19. RoddieIC, Shepherd JT (1957) The effects of carotid artery compression in man with special reference to changes in vascular resistance in the limbs. J Physiol139: 377-384. [Crossref]

20. Barbieri R, Triedman JK, Saul JP (2002) Heart rate control and mechanical cardiopulmonary coupling to assess central volume: a systems analysis. $\mathrm{Am} J$ PhysiolRegulIntegr Comp Physiol283: R1210-1220. [Crossref]

21. Parati G, Grassi G, Coruzzi P, Musiari L, Ravogli A, et al. (1987) Influence of cardiopulmonary receptors on the bradycardic responses to carotid baroreceptor stimulation in man. ClinSci (Lond) 72: 639-645. [Crossref]

Copyright: (92016 Nemoto K. This is an open-access article distributed under the terms of the Creative Commons Attribution License, which permits unrestricted use, distribution, and reproduction in any medium, provided the original author and source are credited. 